\title{
EXPERIENCIA COMUNICACIONAL EN EL DIAGNÓSTICO Y MANEJO ANTENATAL DE NUDO DEL CORDÓN UMBILICAL
}

\author{
Jorge Hasbun H. ${ }^{1}$, Hugo Muñoz C. ${ }^{1}$, Waldo Sepúlveda L. ${ }^{2}$ \\ 1 Departamento de Obstetricia y Ginecología. Hospital Clínico, Universidad de Chile. ${ }^{2}$ Departamento de Obstetricia y \\ Ginecología, Clínica Las Condes.
}

\section{RESUMEN}

Se comunica caso clínico de una madre que consulta en semana 31 de gestación por ausencia de movimientos fetales, y cuyo ultrasonido (US) con escala de grises, Doppler color y tridimensional (3D), demostró imágenes altamente sugerentes de nudo verdadero de cordón umbilical. Se efectuó vigilancia con prueba de movimientos fetales diaria, monitoreo electrónico, hemodinamia fetal y umbilical con doppler, alternados cada tres días, durante siete semanas, demostrando bienestar fetal. Se efectuó operación cesárea cumplida la semana 37 por antecedente de cicatriz uterina previa. El hallazgo intraoperatorio fue una aglutinación de asas del cordón y recién nacido sano. Se discute el diagnóstico falso positivo de nudo por US, el manejo antenatal orientado a la pesquisa oportuna de una eventual oclusión vascular y la experiencia comunicacional con la paciente, que permitió esperar la madurez fetal sin incidentes y con aceptabilidad de la madre, pese al riesgo implícito del diagnóstico presuntivo. Conclusión: En el manejo de alto riesgo se requiere experiencia, apoyo tecnológico y técnicas de comunicación adecuadas con las pacientes, para que una alianza empática permita alcanzar la mayor edad gestacional y la comprensión de un falso positivo.

\section{PALABRAS CLAVE: Cordón umbilical, nudo verdadero, ultrasonido prenatal}

\section{SUMMARY}

We report a case of a pregnant woman who presented with absence of fetal movement at the 31st gestational week. The ultrasound (US) images (gray scale, color Doppler and 3 dimensional) clearly suggested a true knot of the umbilical cord. A close surveillance regime with daily fetal movement test, fetal electronic monitoring and umbilical hemodynamics with Doppler, alternated every three days, was kept for seven weeks, showing fetal wellbeing. At week 37, a cesarean section was performed because of a previous uterine scar. The intraoperative finding confirmed agglutinated and twisted loops, but not a knot of the umbilical cord. The antenatal management is discussed with a close fetal monitoring to detect eventual vascular occlusion in a tight knot and the communication experience with the mother in order to understand and accept a false positive US diagnosis. Conclusion: During a maternal and fetal high risk management is important the professional experience and technological support. On the other hand, the best medical patient communication through an empathetic alliance is required in order to reach a psychological support, longer gestational age and better understanding a false positive test.

\section{KEY WORDS: Umbilical cord, true knot, prenatal ultrasound}




\section{INTRODUCCIÓN}

El nudo verdadero del cordón umbilical es infrecuente, presentando una incidencia de 0,3 a 2,1\% (1), y se presenta habitualmente como hallazgo en el parto. En la mayoría de las observaciones es laxo, sin embargo, se asocia a una mortalidad perinatal aproximada de $6 \%(2,3)$, cuando la constricción del nudo por movimientos fetales o por el descenso de la presentación ocluye la circulación en sus vasos. El diagnóstico antenatal, accesible por imágenes en algunos casos, es aún difícil e incierto, influye en la vigilancia fetal, lo que puede ser determinante de interrupción y genera gran ansiedad en la familia.

El objetivo de esta comunicación es presentar un caso clínico en que la observación de imágenes altamente sugerentes de nudo verdadero hizo plantear el diagnóstico de sospecha, determinando una vigilancia antenatal especial y un manejo comunicacional con la paciente y su familia.

\section{Caso clínico}

Paciente de 32 años, gesta 2 para 0 , antecedente de trombofilia (factor Leyden), en tratamiento con heparina de bajo peso molecular (Fragmin $5000 \mathrm{UI} / \mathrm{d}$ ) y antecedente de miomectomía intramural con entrada a la cavidad uterina.

En la semana 31 consulta por ausencia de movimientos fetales. El examen clínico fue normal y el ultrasonido demuestra un feto vivo, quieto, flujo umbilical arterial normal y crecimiento fetal en percentil 50. Placenta de aspecto ecogénico e inserción normal. La exploración cuidadosa del cordón en escala de grises demostró una gran aglutinación de asas umbilicales en el lago amniótico proyectado en el cuadrante superior derecho del útero, en cuyo centro se identificó imagen similar a la descrita para el diagnóstico de nudo (4) (Figura 1). Al Doppler se observó asas aglutinadas con flujo multidireccional y multicoloreado, con índices de resistencia normales (Figura 2). Se elaboró imagen tridimensional (3D) que confirmó asas con entrecruzamientos perpendiculares entre sí haciendo más sugerente el diagnóstico de nudo verdadero (Figura 3).

Se informó a los padres de esta complicación con el carácter de sospecha a confirmar en el parto, y se indicó una vigilancia antenatal frecuente orientada a pesquisar un eventual compromiso circulatorio y determinar la interrupción del embarazo.

Con el propósito de evitar reacciones emocionales que pudieran interferir una vigilancia de duración no definida, en una situación clínica muy infrecuente, se estableció una cuidadosa relación

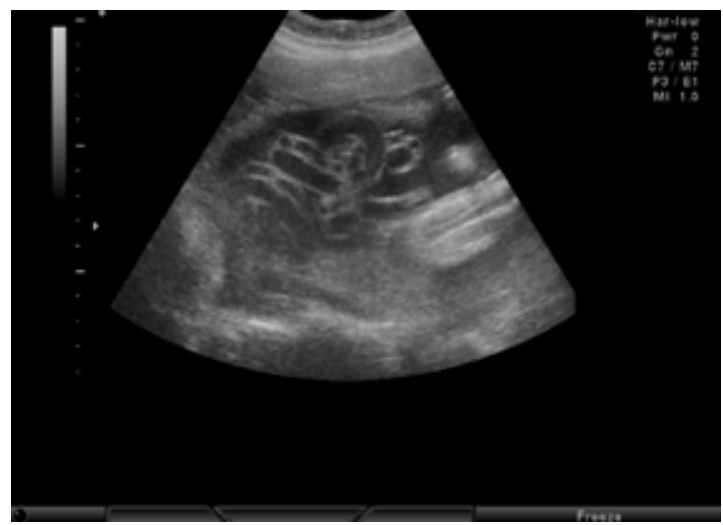

Figura 1. Imagen sugerente de nudo verdadero: asa de cordón rodeando circunferencialmente una sección funicular transversa. Obsérvese aglutinación de asas.

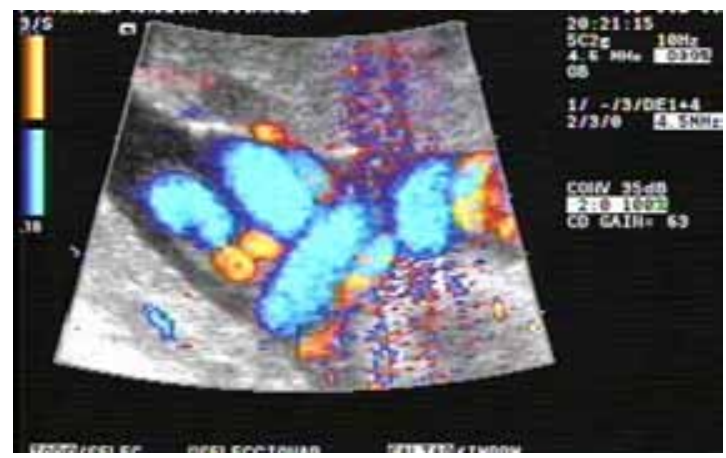

Figura 2. Asas aglutinadas, perpendiculares con flujo multidireccional.

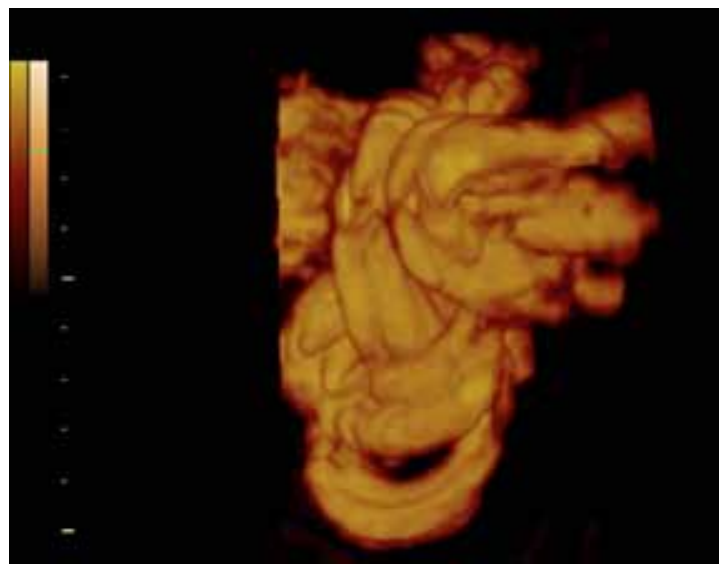

Figura 3. Imagen tridimensional ultrasonido Doppler-poder sugerente de nudo de cordón (falso positivo). 
médico-paciente basada en la guía comunicacional de Calgary-Cambridge (5), la que incorpora elementos de comunicación efectiva permanente en anamnesis, examen físico, diagnóstico, tratamiento y evaluación. Está orientada a satisfacer las necesidades afectivas y de información de la madre, por lo que en el caso se resaltó la seguridad de la vigilancia, los beneficios del cuidado continuo y los daños a evitar.

Los objetivos de la vigilancia fueron evaluar los movimientos del feto como indicador de bienestar, definir periódicamente la reactividad cardiaca, identificar los signos hemodinámicos de redistribución circulatoria y la aparición de pulsaciones en vena umbilical, sugerentes de compresión vascular.

El manejo antenatal incluyó el conteo materno diario postprandial de los movimientos fetales en treinta minutos por tres veces, la exploración Doppler dos veces por semana con evaluación de arteria umbilical, vena umbilical y arteria cerebral media. El monitoreo electrónico de la frecuencia cardiaca fetal se indicó cada siete días y en caso de disminución de los movimientos o inicio de contracciones. Esta vigilancia se mantuvo por siete semanas, confirmando movimientos fetales estables, sin ningún evento hemodinámico de riesgo fetal, en controles que fueron aceptados por la paciente con disposición muy favorable (Figura 4).

Cumplida la semana 37 se procedió a la interrupción del embarazo por cesárea (antecedente de cicatriz uterina). Se obtuvo un recién nacido de 2900 g. Apgar 9-9, adecuado para la edad gestacional y evolución neonatal fisiológica. Placenta de aspecto normal de $310 \mathrm{~g}$. El cordón umbilical estaba dispuesto en el plano horizontal y presentaba aglutinación de asas y al estirarlo no presentaba nudo en su trayecto (Figura 5).

\section{PRUEBA DE MOVIMIENTOS FETALES}

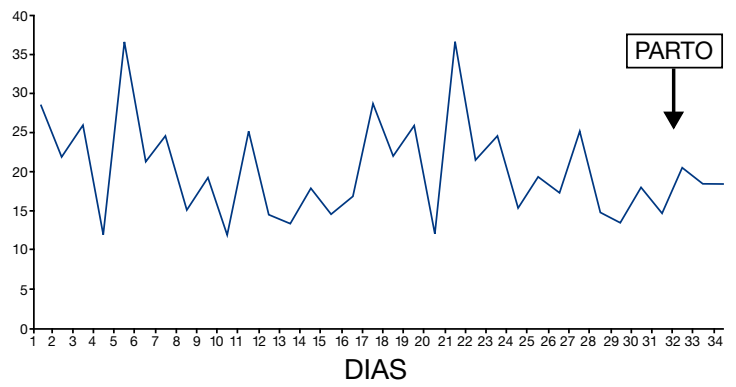

Figura 4. Vigilancia fetal con conteo materno diario de movimientos, monitoreo electrónico y doppler bisemanal.

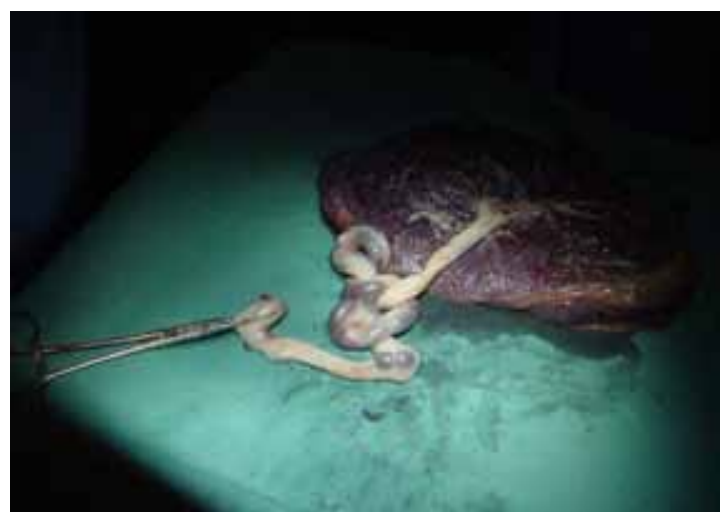

Figura 5. Asas aglutinadas del cordón umbilical, sin nudo.

\section{DISCUSIÓN}

Analizaremos el caso orientado en tres aspectos: el diagnóstico de nudo del cordón umbilical, el manejo antenatal y la experiencia comunicacional. Diagnóstico ultrasónico de nudo: los signos descritos (imagen en hoja de trébol con cuatro hojas, imagen multicoloreada al Doppler color) son inseguros y deben considerarse sugerentes, pero inespecíficos para el diagnóstico $(6,7)$. En el falso positivo, la imagen sospechosa de nudo se origina por aposición de múltiples asas aglutinadas en planos perpendiculares y puede ser transitoria, pero en este caso, fue persistente y sin variaciones en todas las exploraciones del período de observación. En el falso negativo, la limitación es la exploración incompleta del trayecto del cordón y el carácter tridimensional de una estructura flotante desplazable que es observada con ultrasonido bidimensional. López Ramón y Cajal (4), comunica en cinco casos de nudo verdadero, la observación de una imagen de una sección transversa del cordón con sus tres vasos, rodeada por un asa completa y que denomina "hanging noose sign" (Figura 6). La compresión externa y transabdominal del nudo, puede traducirse en la imagen del flujo en la vena umbilical y sería visible en escala de grises y en el Doppler color, siendo esto altamente sugerente del diagnóstico.

En el caso presentado identificamos una imagen similar que fue reforzada por las imágenes del examen con Doppler color, también semejantes a las descritas y que nos llevó a una exploración tridimensional en la que se observó conglomerado de asas, cuya recomposición reveló en su centro, múltiples asas apuestas con ejes perpendiculares y entrecruzadas entre sí, sugiriendo el diagnóstico. En el parto se confirmó la aglutinación observada pero ausencia de nudo. 


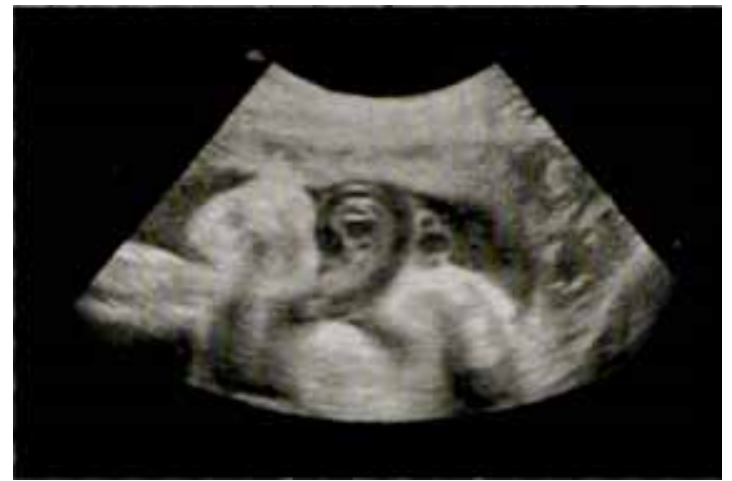

Figura 6. Imagen de nudo (hanging noose sign) descrita por López Ramón y Cajal (referencia 4).

Las imágenes descritas representan un falso positivo, porque la aglomeración de asas puede imitar la imagen de nudo, y su hallazgo debe asumirse con cautela en la información que se entrega a la paciente y también en la conducta, ya que estas imágenes no son determinantes de interrupción del embarazo.

La estrategia de cuidado fetal es necesaria ante la sospecha, ya que por la infrecuencia del problema se desconoce la sensibilidad diagnóstica de las imágenes (verdaderos positivos). Sherer y cols (7), publican un caso de gemelar monocoriónico biamniótico, que por septostomia espontánea se hizo monoamniótico y generó una imagen interpretada como nudo verdadero, que se mantuvo entre las semanas 26 y 34, período en que se efectuó una vigilancia similar a la del caso presentado, confirmando en el parto solo aglutinación de asas. Así, este es el segundo caso de falso positivo que se comunica para el signo descrito.

La evaluación con ultrasonido 3D ha sido utilizada al observar el trayecto continuo de la vena umbilical, en el segmento del cordón comprometido en el probable nudo (8), y su interrupción hace muy probable el diagnóstico. Dado que la confirmación en el parto alcanza solo al $60 \%$ con esta técnica, es necesaria una estrecha vigilancia del crecimiento y bienestar fetal para mantener el embarazo en evolución, o para pesquisar una eventual oclusión vascular y decidir su interrupción. El diagnóstico de nudo verdadero por ultrasonido es posible en algunos casos, aunque éste es difícil, ya que el problema es de muy baja prevalencia y el examen del cordón en toda su longitud consume demasiado tiempo para incluirlo en forma rutinaria.

Hershkovitz y cols (1), identificaron factores de riesgo asociados con nudo (diabetes gestacional, hidroamnios, casos de amniocentesis genética), proponiendo en estos casos exploración dirigida. El diagnóstico también debe sospecharse cuando se detecta compromiso fetal en ausencia de patología materno-fetal. Son necesarios más estudios para precisar el diagnóstico y reducir las complicaciones en esta infrecuente patología.

Manejo antenatal: la presencia o sospecha de nudo verdadero requiere la evaluación fetal con los métodos disponibles, y la conducta puede ser la interrupción del embarazo si se detectan signos de hipoxia, o un manejo conservador si no hay compromiso fetal, de gran valor en casos de edad gestacional temprana.

En nuestro caso, la evaluación fetal se orientó a la detección de un efecto circulatorio de la constricción vascular en el nudo, ya que en estos casos el monitoreo electrónico anormal, la presencia de meconio, el sufrimiento fetal y la muerte fetal son más frecuentes.

Los métodos se utilizaron diariamente (conteo de movimientos fetales) o seriadamente cada tres días (combinando el monitoreo electrónico de la frecuencia cardíaca fetal y la evaluación hemodinámica con Doppler en forma alternada) para asegurar la detección oportuna de un evento hipóxico o su descarte, logrando así la mayor madurez fetal.

La evaluación hemodinámica tuvo dos objetivos: detectar el inicio de hipoxia fetal (demostró IP normales en arterias umbilical y cerebral media en todos los controles) y detectar precozmente un efecto estenótico en el cordón, por la aparición de pulsaciones en vena umbilical transmitidas desde las arterias umbilicales comprimidas por la tracción y con aumento de la velocidad del flujo venoso (9). No fueron visualizadas pulsaciones venosas y la velocidad del flujo permaneció constante en 15 $\mathrm{cm} / \mathrm{s}$, sin la aceleración descrita por efecto estenótico (Figura 7).

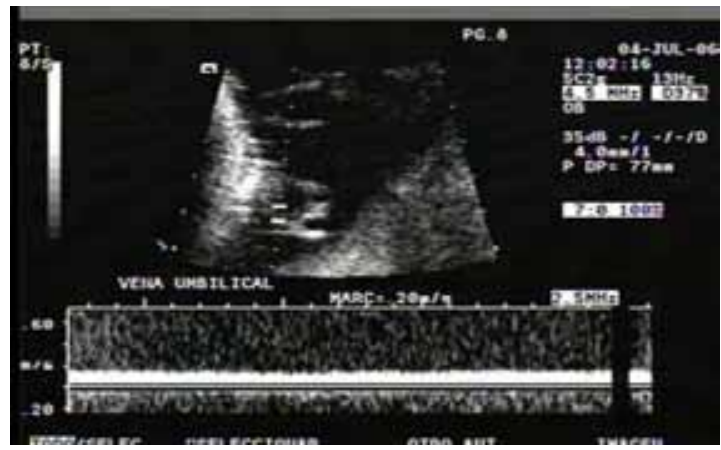

Figura 7. Flujo en vena umbilical sin pulsaciones y velocidad constante $(15 \mathrm{~cm} / \mathrm{s})$. 
La ausencia o disminución de movimientos fetales en período de 12 horas ha demostrado utilidad para reducir a la mitad la muerte fetal $(10,11,12)$. La prueba dio gran tranquilidad a la madre que involucró su propia percepción en el cuidado de su hijo, conocedora que los movimientos obtenidos en cada medición aseguraban el bienestar fetal. Esto generó un contexto de optimismo durante las evaluaciones hemodinámicas. En nuestra opinión el conteo de movimientos fue de gran apoyo a la paciente durante el período de siete semanas en observación con un diagnóstico presuntivo, confirmando la experiencia personal que tenemos con este método $(13,14)$.

En resumen, se efectuaron doce exámenes de Doppler, siete registros basales y recuento materno diario de movimientos. La metodología es discutible en su frecuencia y no orienta a cuál método puede ser más recomendable, pero en conjunto confirmaron un bienestar fetal continuo y dieron seguridad en la conducta médica.

Experiencia comunicacional: en la práctica médica actual existen limitaciones que determinan finalmente insatisfacción en la relación entre pacientes y médicos: sobreuso y subuso de tecnología, manejo inadecuado de la comunicación e información, temores legales, etc. La situación clínica inédita, de incertidumbre diagnóstica y de resultado perinatal, tenía un potencial negativo porque un diagnóstico presuntivo erróneo pudo llevar a una interrupción prematura iatrogénica o a los riesgos de una eventual complicación aguda, surgida durante el período de vigilancia si el diagnóstico presuntivo era correcto. Para la paciente es una situación inconfortable de ansiedad prolongada por la incertidumbre ante los riesgos implícitos. Evitar estos inconvenientes requirió de una alianza médico-paciente empática, alentadora, identificando sus emociones, expectativas y temores. Para ello fueron útiles las técnicas de comunicación apropiadas, considerando las ideas y sentimientos de la paciente y expresando conocimiento y deseo de ayudar.

En el caso presentado, el diagnóstico presuntivo con imágenes fue asumido como probabilidad y no de certeza, decidiéndose con la paciente un manejo de vigilancia basado en los beneficios demostrados con los métodos de evaluación de la unidad feto-placentaria. El diseño de esta vigilancia fue empírico, pero cubriendo con la mayor posibilidad el diagnóstico oportuno de una eventual oclusión funicular.

El diagnóstico y el manejo fueron informados en el contexto de comunicación de riesgos (15), cuyos principios son entregar información en términos sencillos, con entrevistas de duración suficiente y satisfacción de todas las dudas de la paciente y su familia. La información de riesgos fue cualitativa, orientada al caso, evitando los datos numéricos por la escasez de evidencias $(16,17)$, enfatizando las posibilidades de éxito y no el riesgo de muerte o daño. El buen resultado (neonato sano y maduro) fue una meta a obtener en etapas progresivas confirmando el bienestar fetal cada semana, única forma de evitar el error de conducta o la consecuencia de una complicación aguda.

Durante el manejo, exclusivamente ambulatorio, hubo adherencia materna a la vigilancia, con ansiedad controlada y una expectativa optimista, sin necesidad de hospitalización antenatal. Al epílogo se percibió satisfacción familiar y notablemente no hubo ninguna expresión de reparo por la presunción errónea sugerida por las imágenes.

\section{BIBLIOGRAFÍA}

1. Hershkovitz R, Silberstein T, Sheiner E, Shoham I, et al. Risk factors associated with true knots of the umbilical cord. Eur J Obstet Gynecol Reprod Biol 2001;98:36-9.

2. Arias $U$, Heinonen $S$. Clinical significance of true umbilical knots: a population-based analysis. Am J Perinatol 2002;19:127-32.

3. Sherer DM, Anyaegbunam A. Prenatal ultrasonographic morphologic assessment of the umbilical cord. A review. Part II. Obstet Gynecol Surv 1997;52:515-23.

4. Lopez Ramon y Cajal C, Ocampo Martínez R. Prenatal diagnosis of true knot of umbilical cord. Ultrasound Obstet Gynecol 2004;23:99-100.

5. Kurtz SM, Silverman JD, Benson J, Draper J. Marrying content and process in clinical method teaching: enhancing the Calgary-Cambridge Guides. Acad Med 2003;78:802-9.

6. Sepulveda W, Shennan AH, Bower S, Nicolaidis P, Fisk NM. True knot of the umbilical cord: a difficult prenatal ultrasonographic diagnosis. Ultrasound Obstet Gynecol 1995;5:106-8.

7. Sherer DM, Bitton C, Stimphil R, Dalloul M, KhouryCollado F, Abulafia O. Cord entanglement of monochorionic diamniotic twins following spontaneous antepartum septostomy sonographically simulating a true knot of the umbilical cord. Ultrasound Obstet Gynecol 2005;26:676-8.

8. Hasbun J, Alcalde JL, Sepulveda W. Three dimensional power doppler sonography in the prenatal diagnosis of a true knot of the umbilical cord: value and limitations. J Ultrasound Med 2007;1215-20.

9. Gembruch $U$, Baschat AA. True knot of the umbilical cord: transient constrictive effect to the umbilical venous flow demonstrated by doppler sonography. UItrasound Obstet Gynecol 1996;8:53-6.

10. Elbourne D, Grant A. Study results vary in count-to-10 method of fetal movement screening. Am J Obstet Gynecol 1990;163:264-5.

11. Moore TR, Piacquadio K. A prospective evaluation of 
fetal movement screening to reduce the incidence of antepartum fetal death. Study results vary in count to 10 method. Am J Obstet Gynecol 1989;160:1075-80.

12. Sadovsky F. Mothers count fetal activity at least for 30-60 minutes, two or three times daily; movement alarm sign: no movement for 12 hours. Int $\mathrm{J}$ Gynaecol Obtet 1974;12:75-9.

13. Molina R, Hasbun J, Van Cauwelaert R, Thambo S, Nazer J, Fernández P. Transplante renal y embarazo. Tres casos clínicos de donante vivo. Rev Chil Obstet Ginecol 1983;48:238-43.

14. Hasbun J. Experiencia con la Prueba de Movimientos
Fetales en el Consultorio La Pincoya. Comunicación personal (no publicada).

15. Moore P, Rivera S, Corbalán J. Como transmitir la evidencia a los pacientes: El médico como comunicador de riesgo. Rev Med Chile 2006;134:385-90.

16. Bascuñan ML. Cambios en la relación medico-paciente y nivel de satisfacción de los médicos. Rev Med Chile 2005;133:11-6.

17. Edwards A, Elwyn G, Covey J, Mathew E, Pill R. Presenting risk information a review of the effects of framing and others manipulations on patients outcomes. J Health Comm 2001;6:61-82. 